\title{
Development and Application of Biomedical Experiments Teaching Equipment
}

\author{
Hou Yanyang ${ }^{1}$ and Hou Xiaolei ${ }^{1}$ \\ ${ }^{1}$ Zhengzhou university of industrial techonlogy, Henan 451150, China
}

Keywords: DM642; Biomedical Engineering; Comprehensive experiment

\begin{abstract}
In order to meet the experimental teaching of biomedical comprehensive and designing needs. We designed a new type of biomedical teaching experiment system. The equipment features is through PAL CCD HD camera to shoot the CT slice, we use the DSP development board Ethernet port connected to $\mathrm{PC}$, to achieve the exchange of instructions and data transfer between the DSP and PC. We use based on VC++ matching program to control the DSP system. The students could design comprehensive and innovative experiment in the experimental equipment. We can also carry out the curriculum design, graduate design, electronic competition, innovation and entrepreneurship research projects. The application results show that the equipment can deepen students' understanding of the specialized advanced courses and enhance students' practical ability and sense of innovation.

Experiment is an important teaching link of biomedical engineering major and the main practical form of learning biomedical engineering knowledge well. We find that medical image processing, digital signal processing, medical imaging technology, embedded medical instrument design, medical sensor technology, DSP technology and advanced programming language are more difficult in the course of teaching. It is too boring for students to learn, many students can not go deep into the course of learning. Curriculum experiments are mainly confirmatory, students can not really grasp the essence of these courses; the teaching structure also has a lot of unreasonable points, for example, after placing a curriculum with strong comprehensiveness, difficulty and practice, the arrangement of practice links is too little, which affects the development of various innovative activities of students ${ }^{[1-3]}$. Therefore, an experimental teaching device for biomedical specialty based on DM642 is designed.
\end{abstract}

\section{Experimental Installation}

The experimental device (see Fig. 1) includes a 1: pal high definition CCD camera, a DSP circuit board, a PC computer, XDS 510 simulator, a medical watch lamp, a system software, and an Ethernet communication module.

Circuit Board

Figure 2 shows the extended hardware block diagram. The SEED-VPM642 development board (see figure 3) made by Unitas co., Ltd. is used as the main circuit board of the device.

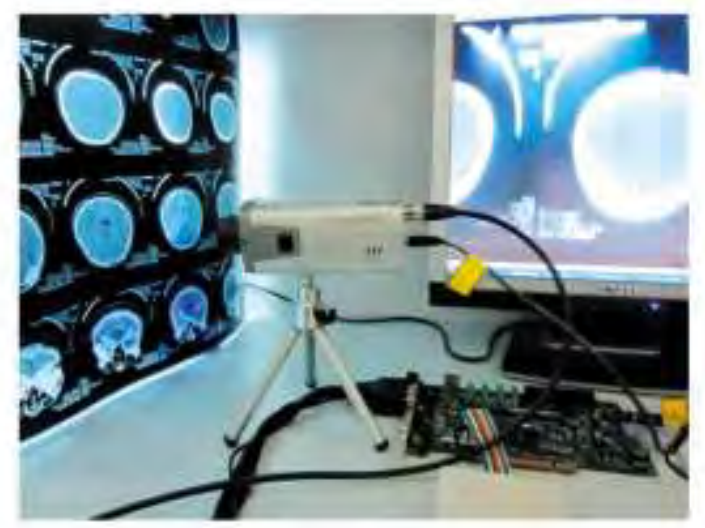

Fig. 1 Experimental Installation

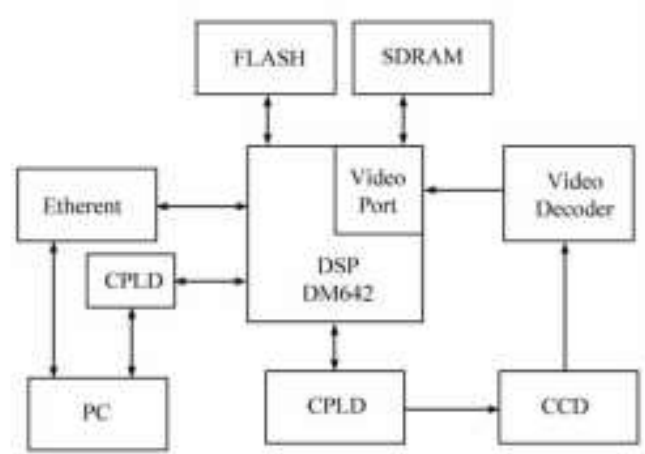

Fig. 2 Hardware system block diagram

SEED-VPM642 development board is a low power development board for all kinds of video applications. Students can develop and apply all kinds of C64xxDSP chips of T1 company.The SEED ${ }^{\wedge}$ VPM642 development board has complete on-board equipment, mainly including the TMS320DM642DSP chip with 
$600 \mathrm{MHz}$ of $\mathrm{T} 1$ company, which has the standard PCI bus structure and can be used as the cooperative processing board; 4 PAL/NTSC video input port and 1 PAL/NTSC video output port; 4 MBX64 bit SDRAM can store up to 32 frames of image; $4 \mathrm{MBX} 8$ bit flash, which can be written into a large number of programs and have the function of self-starting; 2 standard RS232 serial ports can transmit some control commands through the serial port, and a $10 / 100 \mathrm{MB}$ standard Ethernet CPLD chip is used to realize the software configuration of the hardware function of the board by controlling the hardware function of the board by controlling the register inside the CPLD. The video capture module selects CT images collected from the $6.35 \mathrm{~mm}$ (1/4 inch) CCD HD camera produced by Sony and VideoPort decoding module on the DSP development board, CCD supports PAL and NTSC two different analog signal output, Video Port decoding modules are responsible for converting the input analog signal into digital signal for DSP processing. PC adopts Windows XP system, hardware configuration is 1 GB of memory desktop computer. The emulator uses the SEED-XDS510PLUS emulator of Unitas, which supports CCS2.2 and above versions ${ }^{[4-5]}$.

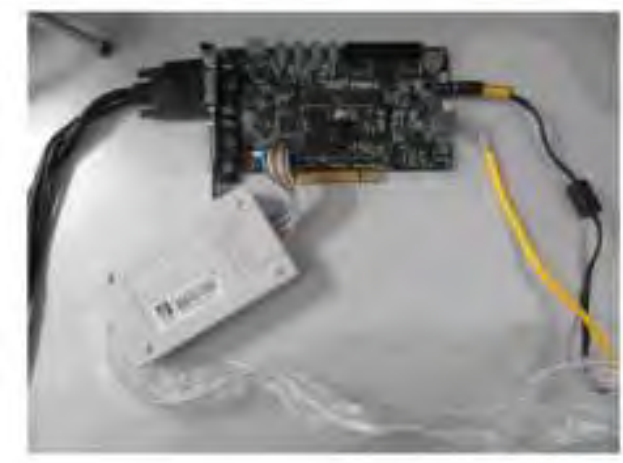

System software

Fig. 3 SEED-VPM642 development board

The system software is divided into DSP on-chip program and PC control system. DSP's on-chip program is generated by CCS(code compos-er Studio software of T1 company. CCS is an integrated development environment based on Windows operating system, which integrates code generation tools and debugging tools, and also provides compilation functions based on visual link and parse, using CCS2.2 Version. The program compiled on the computer is downloaded to the SDRAM of the DSP board by XDS510 simulator. After the PC software issues the command, it calls the corresponding program from the SDRAM according to the command to process the image. Mastering CCS software is a necessary skill for DSP issuers, students can get fully trained on the experimental equipment.

PC control program is written by VC, mainly used to send various commands to DSP and display processing results. The main functions of the software include: (1) Image capture: command DSP to intercept an image; (2) image preprocessing: grayscale conversion, noise filtering and horizontal gradient detection; (3) image enhancement: image smoothing, median filtering, image sharpening; (4) image transformation: image amplification and reduction, Image translation, image horizontal transformation, image vertical transformation, image rotation; (5) edge detection: Sobel edge detection, Kir- Sch edge detection, Laplacian edge detection. In addition, threshold segmentation and restoration of image functions. The software main interface is shown in Figure 4

Ethernet transmission module.

The Ethernet transmission module circuit is shown in figure 5. The EMAC module is the interface between the DSP processor kernel and the off-chip network physical layer data transmission, which is responsible for receiving and transmitting Ethernet data. The Ethernet MAC layer protocol of IEEE802.3 standard is realized by using BCM5221 chip of Broadcom Company. The command transmission and data transmission can be realized through the connection of the physical layer driver HPY) and the RJ-45 interface ${ }^{[6-7]}$.

In the device, the main function of Ethernet port is to receive all kinds of commands from PC and transmit the commands to DSP. At the same time, the communication between DSP on-chip program and PC control program is shown in figure 6.

The RS232 serial port is used to transmit the command while the command is transmitted by the network interface program, which can assist the Ethernet port to control the $\mathrm{DSP}^{[8]}$. 


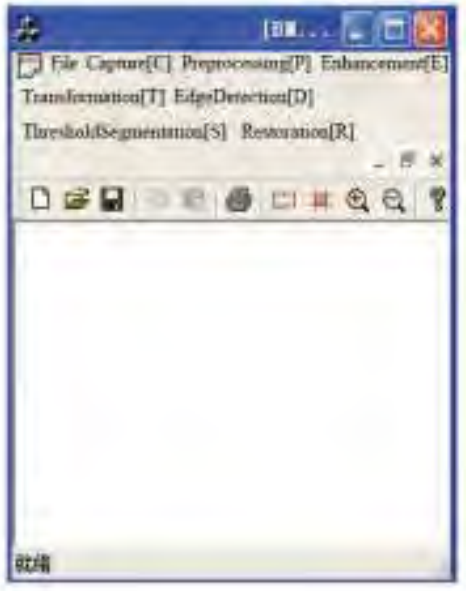

Fig. 4 Software

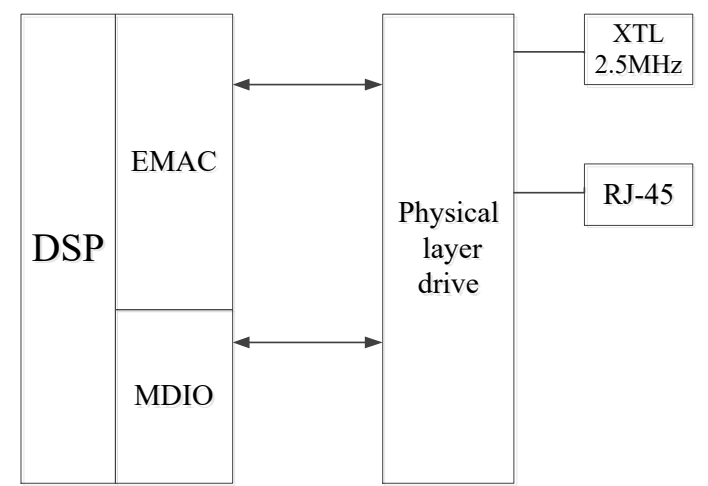

Fig. 5 The Ethernet transmission module circuit

\section{DSP board application

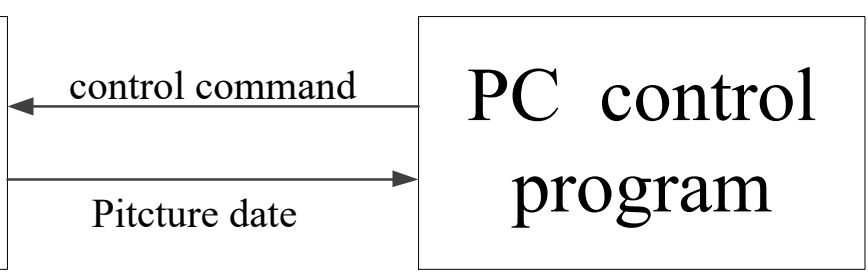

Fig. 6 communication between DSP and PC

\section{Some Functions of Experimental Device}

After starting the experimental device and loading program, you can click on the image menu in the software of PC. (for the menu), see figure 7, DSP will automatically intercept an image in bmp format and pass it to $\mathrm{PC}$, then display it automatically in $\mathrm{VC}$ software.

Find the image inversion option in the image preprocessing menu, see figure 8 . If the current gray scale is $\mathrm{A}$, and the inverse gray scale is $\mathrm{B}$, then the inverse formula is $\mathrm{B}=255-\mathrm{A}$.

Our improved Sobel edge operator ${ }^{[9]}$ is used for edge detection. Figure 9 shows the edge detection interface.

Clicking on the port icon and icon in figure 7 can realize the partial truncation of the picture, click can realize the magnification and reduction of the captured image, and you can also set the zoom multiple in the image transformation to enlarge and shrink the image, as shown in figure 10.

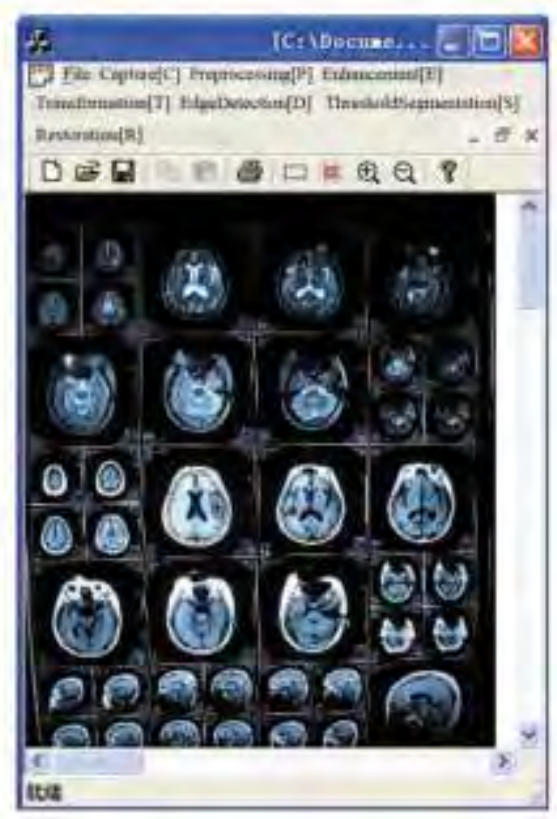

Fig. 7 Intercepted image

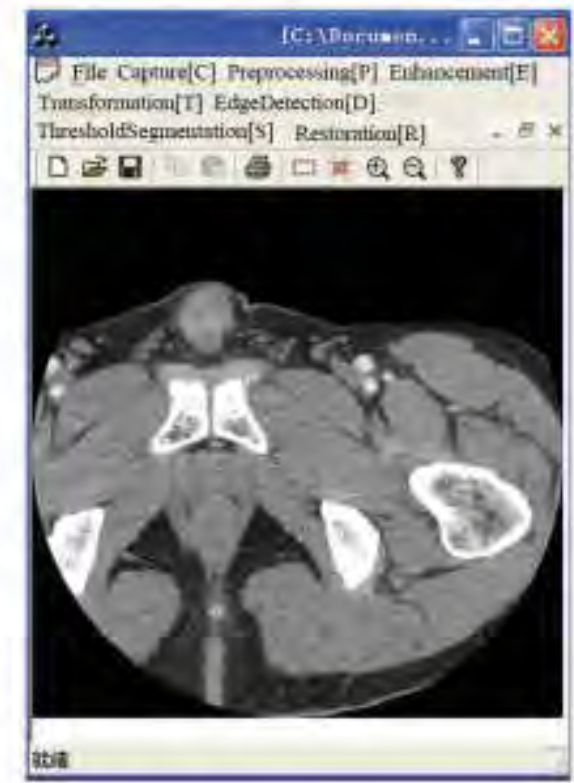

Fig. 8 Image inversion 
Click on the threshold segmentation menu, see figure 11, you can achieve the threshold value of the image segmentation, changing the image to black and white two colors, gray levels are 0 and 255, that is the so-called 'image binarization'.

Limited to space, the above function is only part of the device.

\section{Characteristics of the Device}

The device has the following advantages:

(1) Integration of courses such as medical image processing, medical laboratory and analytical instruments, medical sensor technology, medical imaging technology, and embedded medical device design, DSP technology and advanced programming language. They are closely linked through some comprehensive practical projects, which can strengthen students' practical ability and cultivate students' innovative consciousness.

(2) The contents of the experiment are modularized, systematized, and emphasize the mutual relationship between the theoretical courses. The experimental contents are difficult to combine, which not only retains the original verifiability, but also develops a large number of comprehensive experiments, which can adapt to the teaching needs of students in all grades and all levels.

(3) The course design, graduation plan, electronic competition and innovative entrepreneurial project can also be carried out in this experimental device ${ }^{[10-12]}$.

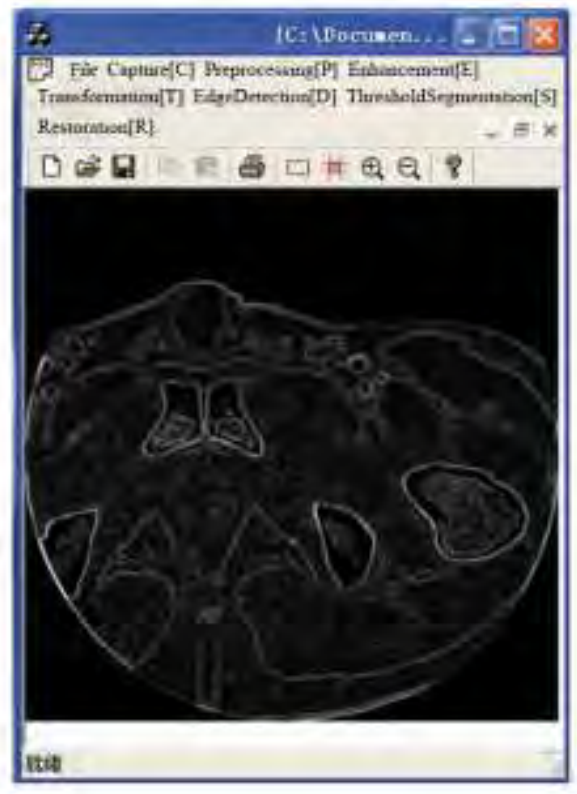

Fig. 9 Edge detection

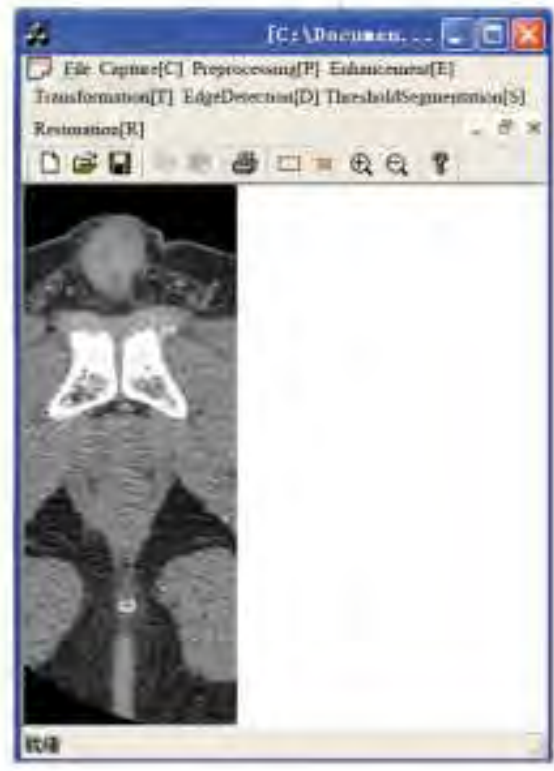

Fig. 10 partial amplification 


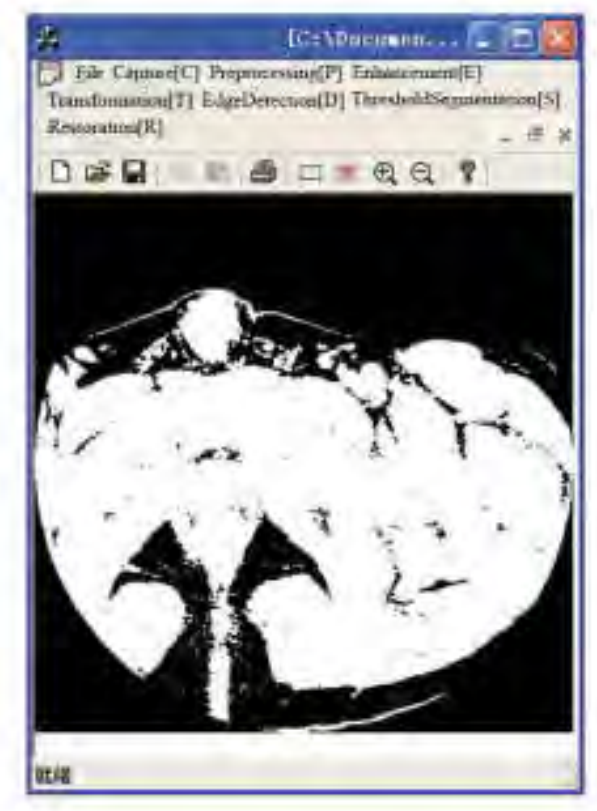

Fig. 11 threshold segmentation

\section{Conclusion}

The device synthesizes the experiments of many courses and solves the original disadvantages of these courses in the teaching method and the course arrangement, so that the students can learn from one another in their study. The device can be used not only for undergraduate experimental teaching of biological medicine, but also for other video related fields.

\section{References}

[1] Feng, B. \& J. Weng. \& N. Huang. 2012. Establishment of Experimental Teaching system for undergraduate Specialty of Biomedical Engineering based on the characteristics of discipline and its own advantages [J]. Experimental Technology and Management, 29(2):68-70.

[2] Ma, C. \& T. Li. \& Z. Li. 2010. Construction of practical Teaching system for Biomedical Engineering [J]. Laboratory research and exploration, 29(4) : 102-106.

[3] Lang, Y. 2011. An improved Sobel Edge Detection operator [J]. Guangxi Light Industry, 5 (5): 59-60.

[4] Pei, T. \& X. Pu. \& G. Niu. 2011. Research on DSP Course Experiment Design Based on Engineering [J]. Experimental Technology and Management, 28(8): 96-99.

[5] Qu, J. 2005. The practice of Comprehensive Design experiment Teaching [J]. Laboratory research and exploration, 24(supplement): 68-81.

[6] Tian, Y. \& W. Liu. \& J. Wang. 2012. Exploration and practice of Comprehensive Design Experimental Project Construction [J]. Experimental Technology and Management, 29(2) :127-129.

[7] Unitas, 2005. SEED-VPM642 User Guide [M]. Beijing: Beijing Unitas Electronic Technology Co., Ltd.

[8] Wang, L. 2010. Face recognition system based on DM642 Development Board [D]. Chengdu, Southwest Traffic University.

[9] Wang, Y. \& J. Liu. 2005. Design and Development of DSP Application system [M]. Beijing: People's Post and Telecommunications Publishing House.

[10] Xie, L. 2006. Realization of Serial Communication between DSP and PC by VC++ [J]. Industrial Control Technology, 6(8): 92-94.

[11] Yuan, L. \& H. Jiao. \& Y. Wang. 2006. Discussion on Biomedical Engineering Teaching from the Development of Biomedical Engineering Industry [J]. Medical Equipment Information, 26(11) : 48-50.

[12] Zhao, J. \& G. Yin. 2006. Digital Image acquisition system based on DM642 [J]. Military automation, 26(5):74, 77. 